\title{
Transient leukocytosis in Emergency Room: an overlooked issue
}

\author{
Giampiera Bertolino, ${ }^{1}$ Federica Quaglia, ${ }^{1}$ Luigia Scudeller, ${ }^{2}$ Iride Ceresa, ${ }^{1}$ Carlo L. Balduini ${ }^{1}$ \\ ${ }^{1}$ Department of Internal Medicine; and ${ }^{2}$ Biometric Unit - Scientific Direction, San Matteo Polyclinic and Scientific Research \\ Foundation, Pavia, Italy
}

\begin{abstract}
Leukocytosis is regarded as a reliable marker of a serious disorder requiring hospitalization. However, leukocytosis often disappears once the patient is admitted to a medical ward; differential diagnosis of leukocytosis is often overlooked in the busy Emergency Room (ER) routine. We retrospectively evaluated the clinical records of 565 consecutive patients admitted to the Department of Internal Medicine (DIM) after examination in ER. Mean leukocyte count was $11.4 \times 10^{9} / \mathrm{L}$ in ER and $10.1 \times 10^{9} / \mathrm{L}$ in DIM $(\mathrm{P}<0.001)$. Leukocytosis was found in $53.1 \%$ of patients in ER, but in $33 \%$ of these it was no longer evident on the following day, unrelated to baseline white blood cells (WBC) count, age, sex, diagnosis, C-reactive protein level and early antibiotic treatment. A reduction in WBC count larger than $40 \%$ from baseline occurred in $13.6 \%$ of all subjects, and in $31.7 \%$ of those with transient leukocytosis. Leukocytosis in ER is frequent, but it is often transient and not associated with an infectious cause. Other causes, including psychological stress caused by the ER access itself, should be considered in the differential diagnosis.
\end{abstract}

\section{Introduction}

Leukocytosis is one of the most commonly encountered laboratory abnormality in clinical medicine and in the majority of cases it derives from an increased number of neutrophils, less frequently of lym-

Correspondence: Giampiera Bertolino, Department of Internal Medicine, Clinical Medicine 3, San Matteo Polyclinic and Scientific Research Foundation, viale Camillo Golgi 19, 27100 Pavia, Italy.

Tel.: +39.0382.501385 - Fax: +39.0382.526284.

E-mail: g.bertolino@smatteo.pv.it

Key words: Leukocytosis; neutrophilia; stress; Emergency Room.

Contributions: GB designed the study and drafted the manuscript; FQ collected the data, helped with interpretation of results and revised the first draft; LS participated in study design, analyzed the data, and revised the manuscript; IC participated in study design and revised the manuscript; CLB participated in study deign and revised the study.

Conflict of interest: the authors do not report any conflict of interest.

Received for publication: 7 April 2016.

Revision received: 31 May 2016.

Accepted for publication: 19 June 2016.

This work is licensed under a Creative Commons Attribution NonCommercial 4.0 License (CC BY-NC 4.0).

(C) Copyright G. Bertolino et al., 2017

Licensee PAGEPress, Italy

Italian Journal of Medicine 2017; 11:41-47

doi:10.4081/itjm.2017.724 phocytes, and even more rarely of monocytes, eosinophils or basophils. ${ }^{1}$ Leukocytosis may represent a physiologic response to infectious and inflammatory trigger, or may be provoked by neoplasia, especially hematological malignancies. ${ }^{2}$ The normal reaction of bone marrow to infection or inflammation leads to an increase in the number of white blood cells, predominantly polymorphonuclear leukocytes and less mature cell forms (the left shift). Physical stress (e.g., from seizures, anesthesia or overexertion) and emotional stress can also elevate white blood cell counts. Medications commonly associated with leukocytosis include corticosteroids, lithium and $\beta$ agonists. Increased eosinophil or basophil counts, resulting from a variety of infections, allergic reactions and other causes, can lead to leukocytosis in some patients. The most common bone marrow disorders can be grouped into acute leukemia, chronic leukemia and myeloproliferative disorders. ${ }^{3}$

Elevated white blood cells (WBC) count is therefore regarded as a reliable indicator of an underlying disease, and blood count is one of the laboratory tests more frequently carried out to diagnose a wide variety of clinical conditions. For instance, leukocytosis in patients with fever is considered as a strong indicator of a serious infection that often requires hospital admission, ${ }^{4}$ while in subjects with abdominal pain it is associated with conditions requiring surgical intervention. ${ }^{5}$ Moreover, leukocytosis is often used to define patients' prognosis: in patients with acute stroke it is related to the area of infarction, ${ }^{6}$ and in subjects with trauma it is a predictor of a worse outcome. ${ }^{7}$

The finding of high WBC count is particularly important in the Emergency Room (ER), where leukocytosis alert to the presence of a serious underlying 
disease and therefore, the hospitalization of subjects who could otherwise be managed as outpatients, might be prompt. On the other hand, it is well known that leukocytosis is not sufficient to define a clinical picture of sepsis, and the clinical gold standard for the diagnosis of bacterial infections is blood culture and not blood count. ${ }^{8}$ Furthermore, thrombocytopenia, lymphocytopenia and bandemia are also predictive of bacteremia. Additional studies have confirmed that bandemia has a high predictive value for the diagnosis of sepsis. ${ }^{9}$

However, in the busy ER routine, patients with leukocytosis and in stable clinical conditions often do not undergo a proper diagnostic workup, antibiotic treatment is prescribed on the basis of Leukocytosis alone (thus potentially in an inappropriate way), while Leukocytosis is not confirmed at subsequent testing. Moreover, except for surgical conditions, current literature does not stress the evidence of leukocytosis in ER as a criterion to hospitalize instead of discharge the patient for a demonstrated prognostic value. Leukocytosis is considered a marker of serious disease in case of extreme leukocytosis (WBC $>25,000 / \mathrm{mmc})^{10}$ or in association with other laboratory findings and patients' characteristics (advanced age, low Glasgow coma scale or mini mental state examination score, hypotension, leukocytosis, metabolic acidosis, and azotemia). ${ }^{11}$

With the present study, we aimed at assessing the proportion of patients with leukocytosis among those first examined in the ER and immediately admitted to a medical ward (as a proxy for stable clinical conditions), to establish the rate of WBC normalization 24 $\mathrm{h}$ after ER assessment, and to screen potential factors associated with normalization of WBC counts.

\section{Materials and Methods}

This retrospective cohort study received ethical clearance by our Institutional Review Board; in our Institution, all patients at admission are asked written informed consent for use of personal and clinical data for research purposes.

We retrospectively included all patients referred to our research and university hospital admitted to the Department of Internal Medicine (DIM) after evaluation in ER from January 2012 to December 2014. Main diagnosis in ER and at discharge from DIM, WBC count in ER and DIM, antibiotic treatments in ER and the first CRP level recorded in DIM were abstracted from clinical charts. Leukocytosis was defined as more than $10 \times 10^{9} \mathrm{WBC} / \mathrm{L}$, neutrophilia as more than $8 \times 10^{9}$ neutrophils/L. The same cell counter was used in each patient for blood cell count in ER and DIM. We defined transient leukocytosis as normal WBC count when assessed in DIM, in patients with leukocytosis in ER.

Descriptive statistics were produced for all vari- ables. Groups were compared with parametric or nonparametric tests, according to data distribution, for continuous variables, and with Pearson's chi-2 test (Fisher exact test where appropriate) for categorical variables. Tests for paired data were used to assess ER-DIM changes within patient. Univariate and multivariate logistic regression models were applied to assess factors associated with transient leukocytosis. In all cases, 2-tailed tests were used. P-value significance cut-off was 0.05 .

\section{Results}

The cohort included 565 patients (Table 1), mainly older adults with a slight predominance of females. As expected in elderly patients, the majority were suffering from cardiopulmonary or cerebrovascular disorders.

Leukocytosis was found in 300 patients $(53.1 \%)$ (Figure 1). The highest prevalence of leukocytosis was observed in systemic infections (76.7\%), but was common also in all the other diagnostic categories (from $16.7 \%$ to $71.4 \%$ ).

On an average of 18 (standard deviation: 5) h later, repeat WBC count normalized in 99 (33\%) of the 300 subjects with leukocytosis in ER. Mean WBC count decreased from 11.4 to $10.1 \times 10^{9} / \mathrm{L}$ in the entire series of patients $\left(11.4 \%\right.$ decrease), from 15.2 to $12.7 \times 10^{9} / \mathrm{L}$ in patients with leukocytosis in ER (16.4\% decrease), and from 13.5 to $8.3 \times 10^{9} / \mathrm{L}(38.6 \%$ decrease $)$ in subjects with transient leukocytosis ( $\mathrm{P}<0.001$ in all cases). Of note, a WBC reduction larger than $40 \%$ was observed in $13.6 \%$ of all subjects with leukocytosis, and in $31.7 \%$ of those with transient leukocytosis (Table 2). As shown in Figure 2, a decrease in WBC count occurred not only in patients with leukocytosis in ER, but also in those with WBC counts lower than $10 \times 10^{9} / \mathrm{L}$.

There was no association between transient leukocytosis and baseline WBC count, age, sex, diagnosis in ER and at discharge from DIM, antibiotic treatment in ER, and C-reactive protein level in DIM (Table 3).

Mean neutrophil count was $9.1 \times 10^{9} / \mathrm{L}$ in ER and $8.1 \times 10^{9} / \mathrm{L}$ in DIM, and the proportion of subjects with neutrophilia decreased from $50 \%$ in ER to $37.5 \%$ in DIM. Of the 300 patients with leukocytosis in ER, 276 (92\%) also had neutrophilia, and this number fell down to 190 (63.3\%) in DIM. As for leukocytosis, also transient neutrophilia was not associated with any patients' characteristics (data not shown).

\section{Discussion and Conclusions}

While differential diagnosis of leukocytosis is taught early in medical schools curricula, in daily clinical practice (in particular in the busy ER routine) it is often overlooked, and antibiotic treatment is often in- 


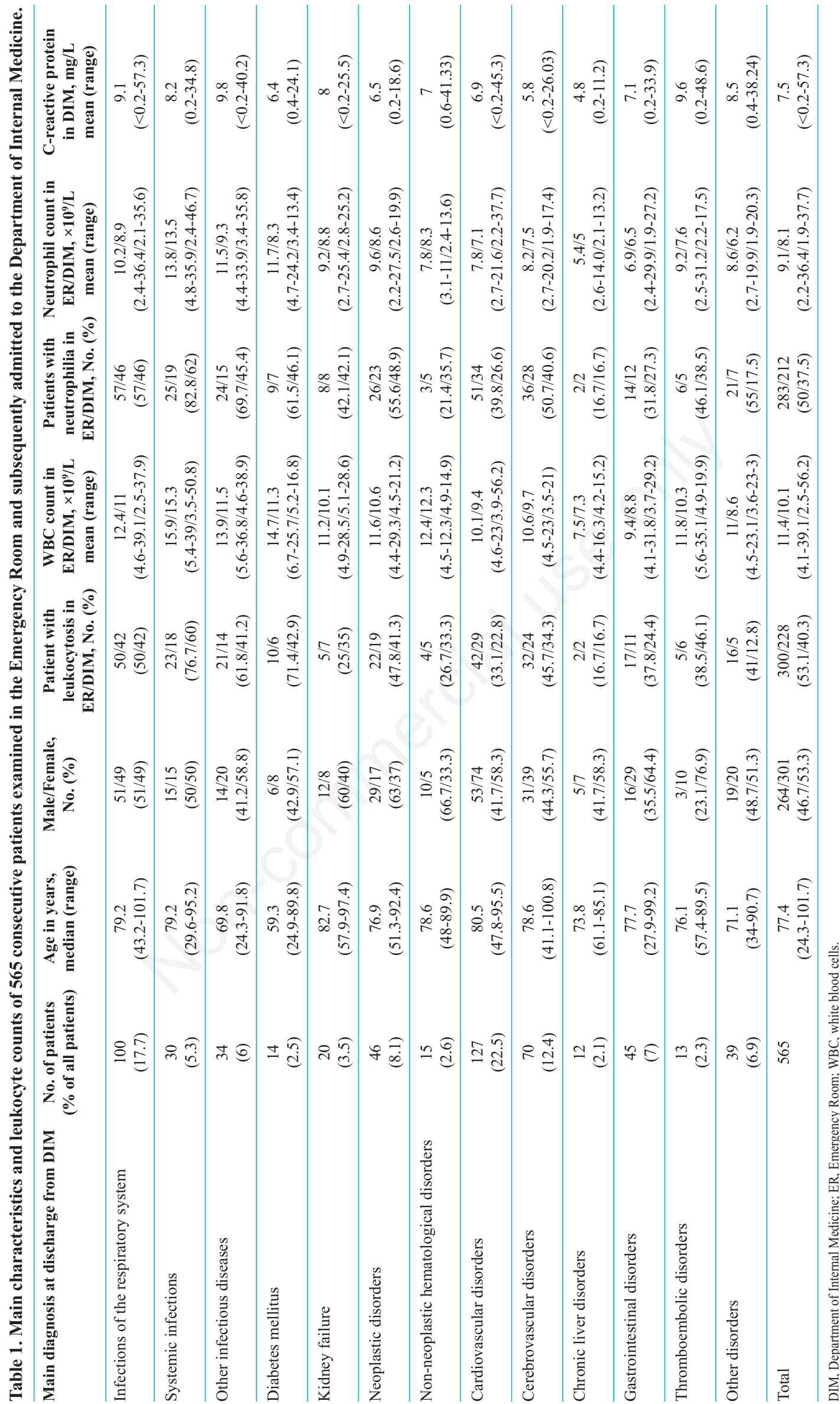


appropriately prescribed without accurately search for other causes of high WBC count.

In our study, on a large cohort of consecutive patients admitted to DIM after ER assessment, we quantify the real-life impact of transient leukocytosis: more than half of the study sample had leukocytosis in ER, but in one third of cases leukocytosis disappeared a few hours later when blood count was repeated in the DIM. The parallel decrease of neutrophil count indicates that the transient leukocytosis observed in ER was due to an increased number of neutrophils.

While neutrophilia is an important indicator of in-

Table 2. Trend of white blood cells count in Emergency Room/Department of Internal Medicine in 300 patients with leukocytosis in Emergency Room.

\begin{tabular}{lccc}
\hline Patients with leukocytosis in ER/DIM (n=300) & Transient leukocytosis & Persistent leukocytosis & Total \\
\hline WBC count in ER/DIM, $\times 10$ /L mean (p25,p75) & $13.55(10.6 ; 14.6) /$ & $16.07(12.2 ; 18) /$ & $15.21(11.5 ; 16.9) /$ \\
& $8.29(7.22 ; 9.5)$ & $14.91(11.6 ; 16.8)$ & $12.68(9.45 ; 14.35)$ \\
\hline Neutrophil count in ER/DIM, $\times 10$ /L mean (p25,p75) & $10.91(8.78 ; 12.10) /$ & $13.34(9.3 ; 15.91) /$ & $12.51(9.14 ; 14.09) /$ \\
& $6.92(5.43 ; 7.55)$ & $12.4(8.93 ; 14.53)$ & $10.52(7.15 ; 12.16)$ \\
\hline Patients with neutrophilia in ER/DIM, No. (\%) & $87.13 / 12.8$ & $92.96 / 89.95$ & $92 / 63.37$ \\
\hline Reduction of leukocytosis $>40 \%(\%)$ & 31.68 & 3.02 & 13.65 \\
\hline
\end{tabular}

ER, Emergency Room; DIM, Department of Internal Medicine; WBC, white blood cells.

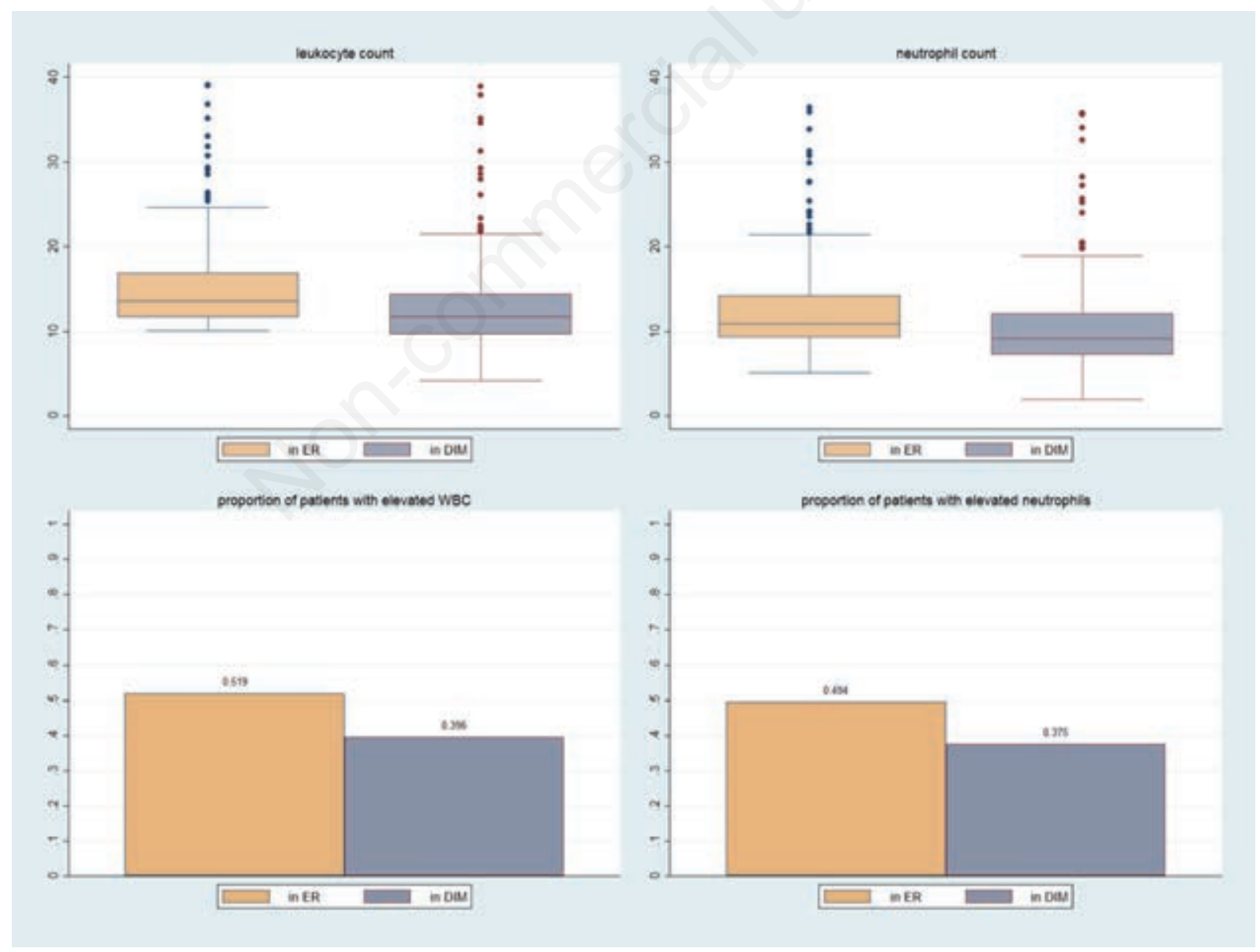

Figure 1. Scatter plot of leukocyte (left) and neutrophil count (right) in the Department of Internal Medicine (DIM) vs Emergency Room (ER), and standard deviation line of the data (which serves as a summary of the data center). WBC, white blood cells. 
fection, transient leukocytosis was not associated with early antibiotic administration and therefore it is unlikely that infection explains all cases of transient leukocytosis.

Moreover, we observed transient leukocytosis also in diagnostic categories other than bacterial infections, either at the presumptive ER diagnosis or the final diagnosis at discharge from DIM. In fact, the rate of leukocytosis was lower in DIM than in ER for all the diagnostic categories, and reduction in leukocyte and neutrophil count occurred not only in subjects with leukocytosis in ER, but also in those with normal WBC values.

Results might be explained (at least in part) by regression to the mean; in fact, we performed a stratified analysis by WBC levels at baseline, and we observed a significant WBC reduction even in patients with normal WBC at baseline.
In our view, these findings indicate that the transient increase in leukocyte and neutrophil counts we observed in ER was a phenomenon affecting all categories of patients independently from any patients' characteristics. Based on these negative results (i.e., lack of association despite adequate sample size) and considering that access to the ER is usually motivated by physical distress, which in turn produces anxiety, we argue that in some cases the transient leukocytosis we observed in this clinical context might be due to psychological stress.

First demonstration that emotional states may be responsible for transient elevation of $\mathrm{WBC}$ has been given in 1926 by Mora et al., ${ }^{12}$ who observed that the number of leukocytes and neutrophils increased by 30 $150 \%$ in dogs placed in front of cats or mice, and by $12-100 \%$ in humans immediately prior to surgery. More recently, an increase in WBC and neutrophils
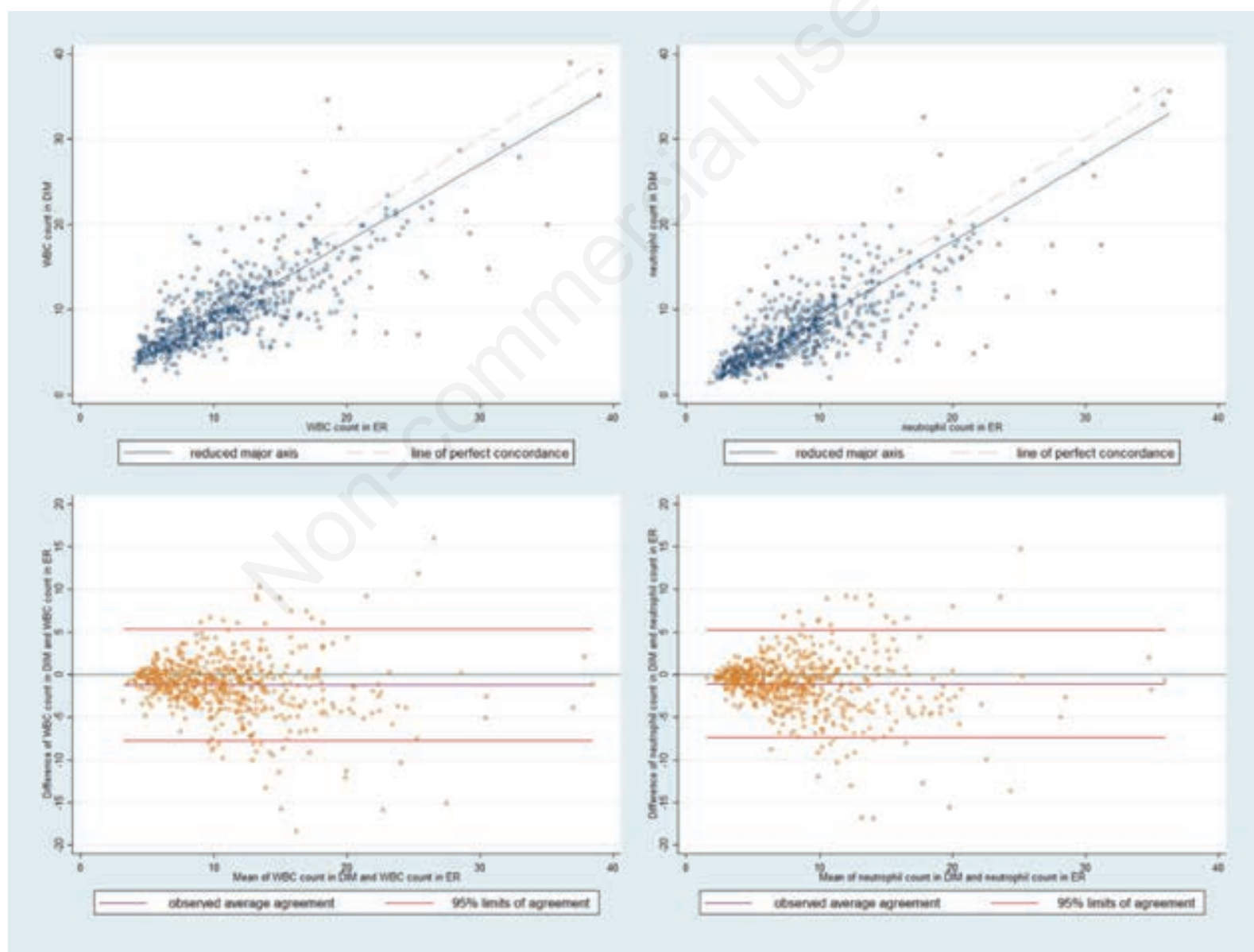

Figure 2. Bland \& Altman plot: data are presented as paired differences plotted against pair-wise means, observed average agreement (i.e., mean difference), and $95 \%$ limits of agreement (i.e., observed average agreement $\pm 1.96 \mathrm{standard}$ deviation), of leukocyte (left) and neutrophil count (right) in the Department of Internal Medicine (DIM) and Emergency Room (ER). The Bland-Altman plot is useful to reveal a relationship between the differences and the magnitude of measurements. In this case, the ample variations between leukocyte and neutrophil counts as measured in ER and DIM seem to be independent from the absolute values of leukocytes and neutrophils. WBC, white blood cells. 
has been observed in healthy subjects waiting for a 15min speaking task ${ }^{13}$ and after sleep deprivation. ${ }^{14}$

Physical stress may also result in leukocytosis. Leukocyte and neutrophil counts increased by more than three times in young people at the end of a strenuous exercise on a cycle ergometer, ${ }^{15}$ and a mean WBC count of $18.9 \times 10^{9} / \mathrm{L}$, with $15.7 \times 10^{9}$ neutrophils/L, was found in 155 athletes after standard marathon. Leukocytes and neutrophils increased by more than two times in a small group of subjects at the end of an ultra-marathon and returned to baseline values $24 \mathrm{~h}$ after. ${ }^{16}$ Also milder physical efforts may significantly increase WBC count, as shown in healthy volunteers after $5 \mathrm{~min}$ of self-paced peak cycle ergometry. In this case, leukocyte counts returned to baseline values in 3 h. ${ }^{17}$

These findings, obtained in well-controlled experimental conditions, suggest that both physical and emotional stress increase WBC count and thus could support our hypothesis.

In fact, the anxiety induced in patients by their illnesses and by an emergency visit is certainly not inferior to the one elicited by the simulation of an exam in healthy subjects. ${ }^{13}$ Similarly, the physical effort required to attend a hospital, especially for elderly individuals, may be well comparable to that of pedaling for $5 \mathrm{~min} .{ }^{17} \mathrm{We}$ hypothesize that, as in experimental stress, leukocytosis might mainly derive from catecholamine-induced demargination of neutrophils, although to prove this hypothesis a prospective design is needed. ${ }^{18}$

The major limitation of this study is its retrospective design, that does not allow us to conclusively prove the pathogenetic mechanisms of transient leukocytosis in ER, since cathecolamine levels (or other markers of stress) at baseline were not available. Also, we were not able to ascertain blood culture results for all patients, and therefore disprove the infectious cause of leukocytosis. ${ }^{8}$ It would be interesting to assess whether transient leukocytosis has an effect on hard outcomes (e.g., hospital mortality, length of hospital stay, etc.) However, in a medical population there are a large number of factors which could confound a potential association between transient leukocytosis and length of hospital stay: in our retrospective analysis, it has not been possible to take them into account.

Further studies are required to better characterize transient leukocytosis, to identify patients most likely to present it in clinical practice and to promptly recognize it, thus preventing inappropriate antibiotic therapy or further unnecessary investigations, benefiting both the health system and the patients themselves.

In conclusion, our study provides a real-life epidemiological quantification of leukocytosis and of

Table 3. Association between patient characteristics and the occurrence of transient leukocytosis.

\begin{tabular}{|c|c|c|c|}
\hline Variable & Odd ratio & Confidence interval & P-value \\
\hline Age & 0.99 & $0.99-1.01$ & 0.322 \\
\hline Female & 0.71 & $0.45-1.14$ & 0.155 \\
\hline C-reactive protein level in DIM & 1.00 & $0.98-1.03$ & 0.825 \\
\hline Antibiotics in ER & 0.54 & $0.25-1.13$ & 0.103 \\
\hline \multicolumn{4}{|l|}{ Main diagnosis at discharge from DIM } \\
\hline Infections of the respiratory system & 0.68 & $0.36-1.29$ & 0.244 \\
\hline Systemic infections & 0.57 & $0.12-2.81$ & 0.494 \\
\hline Other infectious diseases & 1.15 & $0.59-2.22$ & 0.684 \\
\hline Diabetes mellitus & 0.70 & $0.27-1.84$ & 0.471 \\
\hline Hypertension & 1.02 & $0.09-11.37$ & 0.988 \\
\hline Kidney failure & 0.45 & $0.13-1.63$ & 0.227 \\
\hline Cardiovascular disorders & 1.24 & $0.74-2.08$ & 0.416 \\
\hline Neoplastic disorders & 1.27 & $0.51-3.18$ & 0.602 \\
\hline Non-neoplastic hematological disorders & 1.02 & $0.25-4.16$ & 0.978 \\
\hline Cerebrovascular disorders & 0.82 & $0.43-1.58$ & 0.562 \\
\hline Chronic liver disorders & 1.13 & $0.52-2.47$ & 0.749 \\
\hline Gastrointestinal disorders & 1.24 & $0.52-2.94$ & 0.620 \\
\hline Thromboembolic disorders & 0.24 & $0.04-1.33$ & 0.103 \\
\hline
\end{tabular}

DIM, Department of Internal Medicine; ER, Emergency Room. 
transient leukocytosis; transient leukocytosis was often not associated with an infectious cause, and early antibiotic therapy was often inappropriate. Other causes, including psychological or physical stress, should be considered in the differential diagnosis.

\section{References}

1. Chabot-Richards DS, George TI. Leukocytosis. Int J Lab Hematol 2014;36:279-88.

2. Cerny J, Rosmarin AG. Why does my patient have leukocytosis?. Hematol Oncol Clin North Am 2012;26: 303-19.

3. Abramson N, Melton B. Leukocytosis: basic of clinical assessment. Am Fam Physician 2000;1;62:2053-60.

4. Marco CA, Schoenfeld CN, Hansen KN, et al. Fever in geriatric emergency patients: clinical features associated with serious illness. Ann Emerg Med 1995;26:18-24.

5. Potts FE 4th, Vukov LF. Utility of fever and leukocytosis in acute surgical abdomens inoctogenariansand beyond. J Gerontol A Biol Sci Med Sci 1999;54:M55-8.

6. Kammersgaard LP, Jørgensen HS, Nakayama H, et al. Leukocytosis in acute stroke: relation to initial stroke severity, infarct size, and outcome: the Copenhagen Stroke Study. J Stroke Cerebrovasc Dis 1999;8:259-263.

7. Malone DL, Kuhls D, Napolitano LM, et al. Back to basics: validation of the admission systemic inflammatory response syndrome score in predicting outcome in trauma. J Trauma 2001;51:458-463.

8. Marik PE. Don't miss the diagnosis of sepsis! Critical Care 2014;18:529.

9. Cavallazzi R, Bennin CL. Is the band count useful in the diagnosis of infection? An accuracy study in criti- cally ill patients. J Intens Care Med 2010;25:353-7.

10. Lawrence YR, Raveh D. Extreme leukocytosis in the emergency department. Q J Med 2007;100:217-23.

11. Choudhary R, Goel A. Profile of patients hospitalized through the Emergency Room to the Medicine Ward and their short-term outcome at a Tertiary Care Hospital in Delhi. Prehosp Disaster Med 2015 [Epub ahead of print].

12. Mora JM, Altman LE, Hoffman SJ. Effect of mental and emotional states on the leukocyte count. J Am Med Assoc 1926;86:945-6.

13. Goebel MU, Mills PJ, Irwin MR, Ziegler MG. Interleukin-6 and tumor necrosis factor-alpha production after acute psychological stress, exercise, and infused isoproterenol: differential effects and pathways. Psychosom Med 2000;62:591-8.

14. Ricardo JS, Cartner L, Oliver SJ, et al. No effect of a 30 -h period of sleep deprivation on leukocyte trafficking, neutrophil degranulation and saliva IgA responses to exercise. Eur J Appl Physiol 2009;105:499-504.

15. Davison G, Gleeson M. The effect of 2 weeks vitamin C supplementation on immunoendocrine responses to $2.5 \mathrm{~h}$ cycling exercise in man. Eur J Appl Physiol 2006; 97:454-61.

16. Kłapcińska B, Waśkiewicz Z, Chrapusta SJ, et al. Metabolicresponses to a 48-h ultra-marathon run in middleaged male amateur runners. Eur J Appl Physiol 2013; 113:2781-93.

17. Natale VM, Brenner IK, Moldoveanu AI, et al. Effects of three different typesof exercise on blood leukocyte count during and following exercise. Sao Paulo Med J 2003;121:9-14.

18. Benschop RJ, Rodriguez-Feuerhahn M, Schedlowski M. Catecholamine-induced leukocytosis: earlyobservations, current research, and future directions. Brain Behav Immun 1996;10:77-91. 